

\title{
Optimization of Multi-Tool Cutting Modes in Multi-Item Batch Manufacturing System
}

\author{
Genrikh Levin, Boris Rozin, Alexandre Dolgui
}

\section{To cite this version:}

Genrikh Levin, Boris Rozin, Alexandre Dolgui. Optimization of Multi-Tool Cutting Modes in MultiItem Batch Manufacturing System. 7th IFAC Conference on Manufacturing Modelling, Management, and Control, 2013, Jun 2013, St Petersburg, Russia. pp. 766-771, 10.3182/20130619-3-RU3018.00357 . emse-00881533

\section{HAL Id: emse-00881533 \\ https://hal-emse.ccsd.cnrs.fr/emse-00881533}

Submitted on 22 Mar 2019

HAL is a multi-disciplinary open access archive for the deposit and dissemination of scientific research documents, whether they are published or not. The documents may come from teaching and research institutions in France or abroad, or from public or private research centers.
L'archive ouverte pluridisciplinaire HAL, est destinée au dépôt et à la diffusion de documents scientifiques de niveau recherche, publiés ou non, émanant des établissements d'enseignement et de recherche français ou étrangers, des laboratoires publics ou privés. 


\title{
Optimization of Multi-tool Cutting Modes in Multi-item Batch Manufacturing System
}

\author{
G. Levin*, B. Rozin* and A. Dolgui* \\ * United Institute of Informatics Problems \\ Surganov Str, 6, 220012, Minsk, Belarus, (e-mail: \{levin,rozin\}@newman.bas-net.by) \\ ** LIMOS UMR CNRS 6158, Ecole des Mines de Saint-Etienne, \\ 158, Cours Fauriel, 42023 Saint-Etienne cedex, France, (e-mail: dolgui@emse.fr)
}

\begin{abstract}
The problem of optimization of cutting modes for multiple position machines and transfer lines equipped with multi-spindle heads is considered. Parts of several types are grouped into batches. Each tool can be used for machining different parts of a batch. The cutting modes to be defined should provide a required throughput, meet the constraints on the cutting process characteristics and minimize the batch production cost. Decomposition techniques are proposed. These techniques are based on the method of Lagrange multipliers, the concept of fragmentary parameterization and techniques for separable programming problems.
\end{abstract}

Keywords: Process planning, batch manufacturing, cutting modes, multi-spindle heads, machine tools, multi-position transfer lines, optimization.

\section{INTRODUCTION}

The design of production systems is a major issue in industrial engineering and offers a large scope for applications of optimization methods (Dolgui and Proth, 2006).

The determination of cutting modes is a typical optimization problem of manufacturing process design for multi-tool multiple position transfer machines and lines. Appropriate cutting modes increase productivity or reduce manufacturing expenses while providing a required throughput. Cutting modes substantially influence the structure of a manufacturing process, as well as the configuration and parameters of the equipment. In turn, a given structure of manufacturing process and characteristics of equipment impose constraints on the possible values of cutting modes.

Usually, the procedure to determine cutting modes is composed of two phases. First, when designing structure and basic parameters of manufacturing process and equipment, preliminary values of cutting modes are selected. Second, the cutting modes are further optimized taking into account the final structure of the manufacturing process and the parameters of equipment.

Concerning the first phase, in the publications (Dolgui et al., 2006; Dolgui et al., 2008 a,b; Dolgui et al., 2009a,b; Battaia et al., 2012 a,b; Dolgui et al., 2012), mathematical models and methods for optimal design of manufacturing process for multi-position equipment with multi-spindle heads taking into account cutting modes were suggested.

The models and methods for the second phase, dealing with optimization of cutting modes, concern mainly large production series for a single product type (see, for example, Cakir and Gurarda, 2000; Hui et al., 2001; Halevi, 2003; Juan et al., 2003; Levin and Tanaev, 1978; Liang et al., 2001;
Mukherjee and Ray, 2006). With recently growing trends towards large product variety and fluctuations in market demand, building manufacturing systems for a given part family (instead of a sole product) has gained increasing attention (see, for example, Koren et al., 1999, Ismail et al., 2008, Agrawal et al., 2011).

This paper deals with the second phase of machining system design: optimization of cutting modes for given equipment parameters and a fixed structure of manufacturing process. We consider multi-position transfer machines or lines with multi-spindle heads designed for batch manufacturing of different parts in large production series. The special case of this problem was considered by the authors in (Levin et al., 2012). It was supposed that: i) each tool is used to fulfill only identical operations for different parts of a batch, and ii) working stroke for operations executed by the same tool is the same. For this case, decomposition techniques based on the Lagrange's multipliers method, the idea of fragmentary parameterization and dynamical programming approach were proposed. In this new paper, a more general case of the problem is studied where for the same tool cutting process characteristics as well as working strokes can differ when it is used for different parts.

\section{PROBLEM STATEMENT}

The process of multi-tool machining of a sequence of parts composed of identical batches of parts of several types on a multiple position machine or transfer line without buffers is considered.

Working positions of the line are ordered consecutively and each part is machined in this order. The production process is cyclic. In each takt time, all parts are processed simultaneously on their respective positions. Then, all parts are moved simultaneously to their next positions. The part 
from the terminal position is removed and the new part from the input sequence is loaded at the first position. The complete cycle of a batch machining consists of $h$ takt times, where $h$ is the number of parts in a batch. As a result each part of a batch passes (and can be processed) at each working position.

This process is illustrated with the following example for the sequence of parts $(\ldots 2,1,3,1,2,1,3,1,2,1,3,1 \ldots)$ composed of batches $(2,1,3,1)$ that include 4 parts of 3 types each. A part allocation to the working positions of the transfer line for the first takt time is presented in Fig.1. Note that the transfer lines considered are without buffers in between stations.

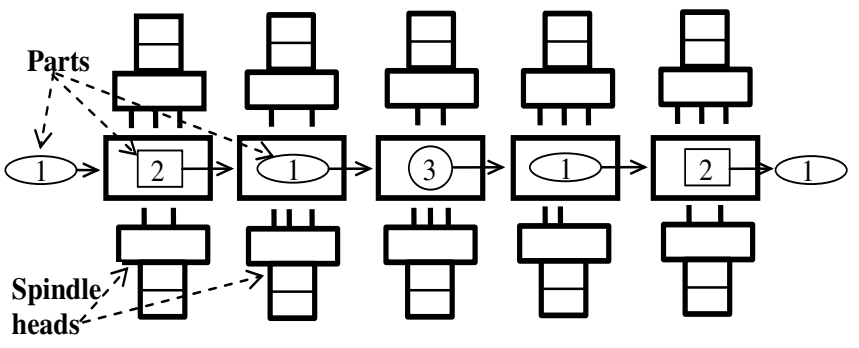

Fig. 1. An example of the allocation of parts to positions

The matrix for the allocation of these parts for all four takt times that compose a complete cycle of batch processing for the considered transfer line is presented in Fig. 2.

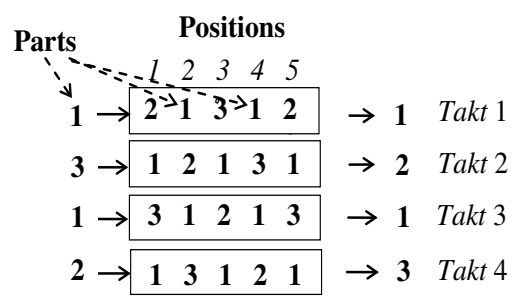

Fig. 2. Allocation of parts to the positions for all takt times of a cycle

The tools of each position are grouped into blocks (tool blocks) of the respective multi-spindle heads. This grouping provides spindle head feeds. The blocks of all working positions and tools of the same block are activated simultaneously. The cutting modes, which are considered here, are the feed per minute for each block and cutting speed for each tool. These modes are defined for each part of a batch. The following is assumed to be known: i) the ranges of possible values of cutting modes, ii) dependences of the tool life time and characteristics of the cutting process on cutting modes, finally iii) the maximal possible values of these characteristics for all tools and tool blocks.

It is also assumed that each tool can be used to machine different parts of a batch in different takt times. The parameters of manufacturing operations (such as cutting depth, diameter, cutting length, material type, etc.) can differ for different parts. Since blocks of tools can also differ from each other for different parts, then some spindle heads may need to be changed before processing the next part or rotary spindle heads should be used. Note that reconfigurable machines can be also used here. Some problems and approaches to application of reconfigurable machines for such machining processes are discussed in (Koren, 2010).

The problem is to determine the cutting modes minimizing the production cost of a batch while obtaining the required throughput under given technological constraints. These constraints take into account ranges of possible values for the cutting modes for each tool and part, as well as limiting values of considered physical and technical characteristics of the machining process for each pair "part-tool" (such as force, rotational moment, roughness, etc.) and for each pair "parttool block" (such as total force and power).

\section{MATHEMATICAL MODEL FORMULATION}

3.1. Basic input data necessary to formulate a mathematical model of the problem in question are: specifications of the parts to be machined, required throughput, equipment parameters, machining process parameters and limiting values for the process characteristics considered.

The following notations will be used below for the input data:

$\mathbf{M}=\{1, \ldots, m\}$ is a set of part types in a batch;

$h_{d}$ is a number of parts of type $d \in \mathbf{M}$ in a batch;

$h=\sum_{d=1}^{m} h_{d}$ is a total number of parts in a batch, $h \geq m ;$

$\delta=\left\{\delta_{1}, \ldots, \delta_{h}\right\}$ is a sequence of parts in a batch, $\delta_{r} \in \mathbf{M}$, $r=1, \ldots, h$;

$n$ is a number of working positions;

$\mathbf{I}_{k}$ is a set of tool blocks at the position $k=1, \ldots, n$, $\mathbf{I}_{k^{\prime}} \cap \mathbf{I}_{k^{\prime \prime}}=\varnothing$ for any $k^{\prime}, k^{\prime \prime}=1, \ldots, n, k^{\prime} \neq k^{\prime \prime} ;$

$\mathbf{I}_{k d}$ is a subset of tool blocks from the set $\mathbf{I}_{k}$ that works up the part $d \in \mathbf{M}$;

$\mathbf{J}_{i}$ is a set of tools of the block $i \in \mathbf{I} \bigcup_{k=1}^{n} \mathrm{I}_{k}, \mathbf{J}_{i^{\prime}} \cap \mathbf{J}_{i^{\prime \prime}} \varnothing$ for any $i^{\prime}, i^{\prime \prime} \in \mathbf{I}, i^{\prime} \neq i^{\prime \prime}$;

$\mathbf{J}_{i d}$ is a subset of tools from the set $\mathbf{J}_{i}$ that works up the part $d \in \mathbf{M}, \bigcup_{d \in \mathbf{M}} \mathbf{J}_{i d} \quad \mathbf{J}_{i} ;$

$l_{i j d}$ is a cutting length in machining part $d \in \mathbf{M}$ with the tool $j \in \mathbf{J}_{i d}$;

$L_{i d}$ is a working stroke of tool block $i \in \mathbf{I}$ in machining the part $d \in \mathbf{M}$;

$\left[\underline{S}_{i d}, \bar{S}_{i d}\right]$ is a range of possible values of the feed per minute for tool block $i \in \mathbf{I}$ in machining part $d \in \mathbf{M}$;

$\left[\underline{w}_{i j d}, \bar{w}_{i j d}\right]$ and $\left[\underline{s}_{i j d}, \bar{s}_{i j d}\right]$ are ranges of possible values of cutting speed and feed per revolution for tool $j \in \mathbf{J}_{i}$ in machining part $d \in \mathbf{M}$;

$\mathbf{P}_{i j d}$ and $\mathbf{P}_{i d}$ are sets of indexes of accountable process characteristics for tool $j \in \mathbf{J}_{i}$ in machining part $d \in \mathbf{M}$;

$\bar{R}_{p i j d}$ and $\bar{R}_{p i d}$ are maximal possible values of characteristics $p \in \mathbf{P}_{i j d}$ and $p \in \mathbf{P}_{i d}$;

$t_{0}$ is a maximal possible value of the portion of batch production time depending on cutting modes; 
Similarly to (Levin and Tanaev, 1978), the expected life time $T_{i j d}(S, w)$ for tool $j \in \mathbf{J}_{j d}$ is calculated as a function of feed per minute $S$ and cutting speed $w$, as follows:

$$
T_{i j d}(S, w) \min _{u=1, \bar{u}_{i j d}} C_{i j u d}^{\prime} /\left(S^{\eta_{i j u d}^{\prime}} w^{\mu_{i j u d}^{\prime}}+G_{i j u d}^{\prime}\right) .
$$

The machining process characteristics $R_{p i j d}(S, w)$ for $p \in \mathbf{P}_{i j d}$ are approximated with well known Taylor's functions:

$$
R_{p i j d}(S, w) \quad C_{p i j d} S^{\alpha} p i j d w^{\beta} p i j d,
$$

In (1) and (2), parameters $C_{i j d}^{\prime}, \eta_{i j d}^{\prime}, \mu_{i j d}^{\prime}, G_{i j d}^{\prime}, \bar{u}_{i j d}, C_{p i j d}$, $\alpha_{\text {pijd }}, \beta_{\text {pijd }}$ are assumed to be given.

Later on:

$\mathbf{M}_{i j}=\left\{d \in \mathbf{M} \mid j \in \mathbf{J}_{i d}\right\}$ is a set of parts worked up with the tool $j \in \mathbf{J}_{i}$;

$\mathbf{M}_{i}=\bigcup_{j \in \mathbf{J}_{i}} \mathbf{M}_{i j}$ is a set of parts worked up with tool block $i \in \mathbf{I}$.

3.2. Parameters of cutting modes to be defined are feed per minute and cutting speed for each tool. It is assumed that: (i) these parameters remain invariable and (ii) the feed per minute is the same for all tools of the same block when identical parts are machined, but the value of this feed can differ for different part types.

To divide a set $\mathbf{M}_{i}$ into subsets of parts (with the same feed per minute when machining them with tools of block $i \in \mathbf{I}$ ), a graph $G_{i}\left(\mathbf{M}_{i}, V_{i}\right)$ is considered, where $\left(d_{p}, d_{s}\right) \in V_{i}$ for $d_{p}, d_{s} \in \mathbf{M}_{i}$ if and only if $\mathbf{J}_{i d_{p}} \cap \mathbf{J}_{i d_{s}} \neq \varnothing$. Let this graph have $\gamma_{i}$ connectivity components $G_{i}^{\vartheta}=\left(\mathbf{M}_{i}^{\vartheta}, V_{i}^{\vartheta}\right), \vartheta=1,2, \ldots, \gamma_{i}$. The feed per minute of block $i \in \mathbf{I}$ must be the same for machining any part from subset $\mathbf{M}_{i}^{\vartheta}, \vartheta=1,2, \ldots, \gamma_{i}$. It is relatively easy to show that $\mathbf{J}_{i}^{\vartheta^{\prime}} \cap \mathbf{J}_{i}^{\vartheta^{\prime \prime}}=\varnothing$ for any $\vartheta^{\prime}, \vartheta^{\prime \prime}=1,2, \ldots, \gamma_{i}, \quad \vartheta^{\prime} \neq \vartheta^{\prime \prime}$, and $\bigcup_{\vartheta=1}^{\gamma_{i}} \mathbf{J}_{i}^{\vartheta} \quad \mathbf{J}_{i}$, where $\mathbf{J}_{i}^{\vartheta} \quad \bigcup_{d \in \mathbf{M}_{i}^{\vartheta}} \mathbf{J}_{i d}$.

So all variables of the cutting modes to be defined can be divided into the following groups according to subsets of parts $\mathrm{M}_{i}^{\vartheta}$ for each tool block $i$ : feed per minute $S_{i}^{\vartheta}$ for tool block $i$ and cutting speed $w_{i j}$ for tool $j$ for all $i \in \mathbf{I}, j \in \mathbf{J}_{i}^{9}$, $\vartheta=1,2, \ldots, \gamma_{i}$.

Later, the following additional notations will be used: $\vartheta_{i j}$ is an index of the set $\mathbf{M}_{i}^{\vartheta}$ that $\mathbf{M}_{i j} \subseteq \mathbf{M}_{i}^{\vartheta}$;

$\vartheta_{i}(d)$ is an index of the set $\mathbf{M}_{i}^{\vartheta}$ that contains the part $d \in \mathbf{M}_{i}$;

$x_{i j} \quad\left(S_{i}^{\vartheta_{i j}}, w_{i j}\right), X_{i}^{\vartheta} \quad\left(S_{i}^{\vartheta},\left(w_{i j} \mid j \in \mathbf{J}_{i}^{\vartheta}\right)\right)$, and

$X=\left(X_{i}^{\vartheta} \mid i \in \mathbf{I}, \vartheta=1, \ldots, \gamma_{i}\right)$.

3.3. Productivity and cost for the process. The general view of the variable portions of cost and time expenses for production of one batch depending on the cutting modes can be expressed as follows:

$\Theta_{1}(X) \quad E_{1} t(X)+Q_{1}(X)$,

$\Theta_{2}(X) \quad E_{2} t(X)+Q_{2}(X)$,

where: $t(X)$ is the portion of the time spent for production of batch $\delta$ which depends on the cutting modes; $Q_{1}(X)$ and $Q_{2}(X)$ are portions of expenses on tooling and of time spent for tools replacement per batch, respectively; $E_{1}$ and $E_{2}$ are expenses and auxiliary time per unit of time when equipment is functioning, respectively.

Since the machining process consists of cyclically repeated identical subsequences of $h$ takt times, so it is sufficient to take into account only one such subsequence. Without loss of generality it is assumed that $\mathbf{H}=\{1, \ldots, r, \ldots, h\}$ is such a subsequence of takt times, and in takt time $r=1$ at working position $k=1$, part $\delta_{1}$ of the batch is worked up. Thus, in takt time $r \in \mathbf{H}$ at position $k \in\{1, \ldots, n\}$, part $d(k, r)=\delta_{\chi(k, r)} \in \mathbf{M}$ is machined, where $\chi(k, r)=1+\bmod ((h+r-\bmod (k, h)), h)$.

For fixed $X$, the variable portion of the duration of batch $\delta$ processing is $t(X) \sum_{r=1}^{h} t_{r}(X)$, where $t_{r}(X)=\max \left\{L_{i d(k, r)} / S_{i}^{9}\right.$ । $\left.k=1, \ldots, n ; i \in \mathbf{I}_{k d(k, r)}, d(k, r) \in \mathbf{M}_{i}^{9}\right\}$ is the variable portion of duration of $r$-th takt time.

Later $\overline{\mathbf{t}}(X)=\left(t_{r}(X) \mid r=1, \ldots, h\right)$.

In this paper, it is assumed that tools are replaced separately after machining a given number of parts. This threshold number for each tool is defined taking into account expected life time with respect to cutting modes.

In accordance with (1), the maximal number of parts $d \in \mathbf{M}_{i j}$ machined with tool $j \in \mathbf{J}_{i}$ during its expected life time $T_{i j d}\left(x_{i j}\right)$ under fixed $X$ is approximated as follows:

$$
D_{i j d}\left(x_{i j}\right)=T_{i j d}\left(x_{i j}\right) S / l_{i j d}=1 / \max _{u=1, \bar{u}} b_{i j d} b_{i j u d}\left(S^{\eta_{i j u d}} w^{\mu_{i j u d}}+G_{i j u d} / S\right),
$$

where $\quad(S, w)=x_{i j}, \quad$ parameters $\quad b_{i j u d}, \eta_{i j u d}, \mu_{i j u d}, G_{i j u d} \quad$ are calculated by using $l_{i j d}$ and parameters from (1).

With abovementioned assumptions, the number of batches machined by tool $j \in \mathbf{J}_{i}$ during its expected life time is: $D_{i j}\left(x_{i j}\right)=\left(\sum_{d \in \mathbf{M}_{i j}} h_{d} / D_{i j d}\left(x_{i j}\right)\right)^{-1}$.

Thus, $Q_{1}(X) \quad \sum_{i \in \mathbf{I}} \sum_{j \in \mathbf{J}_{i}} g_{1 i j} / D_{i j}\left(x_{i j}\right), \quad Q_{2}(X) \quad \sum_{i \in \mathbf{I}} \sum_{j \in \mathbf{J}_{i}} g_{2 i j} / D_{i j}\left(x_{i j}\right)$, where $g_{1 i j}$ and $g_{2 i j}$ are respectively expenses and time spent for one replacement of tool $j \in \mathbf{J}_{i}$.

3.4. Ranges of feasible values of cutting modes can be represented in the following way:

- $\left[\underline{S}_{i}^{\vartheta}, \bar{S}_{i}^{\vartheta}\right]=\left[\max \left\{\underline{S}_{i d} \mid d \in \mathbf{M}_{i}^{\vartheta}\right\}, \min \left\{\bar{S}_{i d} \mid d \in \mathbf{M}_{i}^{\vartheta}\right\}\right]$ is a range of feasible values for parameter $S_{i}^{\vartheta}, \quad i \in \mathbf{I}, \vartheta=1, \ldots, \gamma_{i}$; 
- $\left[\underline{w}_{i j}, \bar{w}_{i j}\right]=\left[\max \left\{\underline{w}_{i j d} \mid d \in \mathbf{M}_{i j}\right\}, \min \left\{\bar{w}_{i j d} \mid d \in \mathbf{M}_{i j}\right\}\right]$ is a range of feasible values for parameter $w_{i j}, i \in \mathbf{I}, j \in \mathbf{J}_{i}$;

- $\left[\underline{s}_{i j}, \bar{s}_{i j}\right]=\left[\max \left\{\underline{s}_{i j d} \mid d \in \mathrm{M}_{i j}\right\}, \min \left\{\bar{s}_{i j d} \mid d \in \mathrm{M}_{i j}\right\}\right]$ is a range of feasible values for feed per revolution $S_{i}^{\vartheta} / w_{i j}, i \in \mathbf{I}, \vartheta=1, \ldots, \gamma_{i}$, $j \in \mathbf{J}_{i}^{\vartheta}$.

Then, the mathematical model for determining optimal cutting modes $X^{*}$ can be formulated as follows:

$$
\begin{aligned}
& \Theta_{1}(X) \rightarrow \min ; \\
& \Theta_{2}(X) \leq t_{0} \text {; } \\
& \underline{S}_{i}^{\vartheta} \leq S_{i}^{\vartheta} \leq \bar{S}_{i}^{\vartheta}, \vartheta=1, \ldots, \gamma_{i}, i \in \mathbf{I} ; \\
& \underline{w}_{i j} \leq w_{i j} \leq \bar{w}_{i j}, j \in \mathbf{J}_{i}, i \in \mathbf{I} \text {; } \\
& \underline{s}_{i j} \leq S_{i}^{\vartheta} / w_{i j} \leq \bar{s}_{i j}, i \in \mathbf{I}, \vartheta=1, \ldots, \gamma_{i}, j \in \mathbf{J}_{i}^{\vartheta} ; \\
& R_{p i j d}\left(x_{i j}\right)=C_{p i j d}\left(S_{i}^{\vartheta}\right)^{\alpha} p i j d w_{i j}^{\beta}{ }^{p i j d} \leq \bar{R}_{p i j d}, i \in \mathbf{I}, \vartheta=\mathbf{1}, \ldots, \gamma_{i}, \\
& j \in \mathbf{J}_{i}^{\vartheta}, d \in \mathbf{M}_{i j}, p \in \mathbf{P}_{i j d} ; \\
& R_{p i d}\left(X_{i}^{\vartheta}\right) \quad \sum_{j \in \mathbf{J}_{i d}} R_{p i j d}\left(x_{i j}\right) \leq \bar{R}_{p i d}, i \in \mathbf{I}, \vartheta=\mathbf{1}, \ldots, \gamma_{i}, d \in \mathbf{M}_{i}^{\vartheta} \text {, } \\
& p \in \mathbf{P}_{i d} \text {. (7) }
\end{aligned}
$$

The aim is to minimize the production cost (1); constraint (2) provides the required throughput; constraints (3)-(7) give the set machining conditions. Problem (1)-(7) will be now referred to as problem A.

Problem $\mathbf{A}$ is a complex nonlinear program. It can be transformed into a posinomial geometrical programming or convex programming problem. In the next section, a special method to solve problem $\mathbf{A}$ is proposed. The method exploits some of its peculiarities.

\section{SOLUTION TECHNIQUE}

In the method proposed, a combination of the Lagrange multipliers and fragmentary parameterization concepts as well as some techniques for separable convex programming problems is used. This is an extension of previous results for the simple case when $m=h=1$ (Levin and Tanaev, 1978) to the more general case when $1<m \leq h$.

Let $\mathbf{X}_{i}^{\vartheta}$ and $\mathbf{X}$ be the sets of $X_{i}^{\vartheta}$ and $X$ that meet the constraints (3)-(7). Let $X^{*}(\lambda)$ be the value of $X \in \mathbf{X}$ that minimizes Lagrange function $L(X, \lambda)=\lambda \Theta_{1}(X)+(1-\lambda) \Theta_{2}(X)$ for a fixed $\lambda \in[0,1]$ (sub-problem $\mathbf{A}_{1}(\lambda)$ ).

It is obvious that if $\Theta_{2}\left(X^{*}(0)\right)>t_{0}$, then constraints (2)-(7) are incompatible, if $\Theta_{2}\left(X^{*}(1)\right) \leq t_{0}$, then $X^{*}(1)$ is a solution $X^{*}$ of problem A. It can be shown that otherwise, $X^{*} \quad X^{*}\left(\lambda^{*}\right)$ where $\lambda^{*} \in(0,1)$ is a root of the equation $\Theta_{2}\left(X^{*}(\lambda)\right)=t_{0}$ with the monotonic function in its left side (sub-problem $\mathbf{C}_{1}$ ). In this case, the problem $\mathbf{A}$ can be solved with the following twolevel decomposition scheme:

- at the lower level, the sub-problem $\mathbf{A}_{1}(\lambda)$ to determine $X^{*}(\lambda)$ is solved for a fixed $\lambda: L(X, \lambda) \rightarrow \min , X \in \mathbf{X}$;
- at the upper level, the sub-problem $\mathbf{C}_{1}$ to find $\lambda^{*}$ is solved using the well-known methods for finding the roots of equations.

Problem $\mathbf{A}_{1}(\lambda)$ is substituted with the equivalent parameterized problem $\mathbf{B}_{1}(\lambda)$ by means of the following transformations: (i) replacement of components $t_{r}(X)$ in the objective function (1) and constraint (2) with parameters $T_{r}$; (ii) supplementation of a set $\overline{\mathbf{T}}_{0}$ of possible values of vector $T\left(T_{1}, \ldots, T_{h}\right)$ of these parameters; and (iii) adding constraints that take into account interrelation of these parameters with cutting modes $X$.

The set $\overline{\mathbf{T}}_{0}$ should include set $\{\overline{\mathbf{t}}(X) \mid X \in \mathbf{X}\}$, in particular it can be assumed $\quad \overline{\mathbf{T}}_{0} \prod_{r \in \mathbf{H}} \overline{\mathbf{T}}_{0 r}, \quad$ where $\quad \overline{\mathbf{T}}_{0 r}=\left[\underline{\mathbf{T}}_{r}, \overline{\mathbf{T}}_{r}\right]$, $\underline{\mathbf{T}}_{r}=\max \left\{L_{i d(k, r)} / \bar{S}_{i}^{\vartheta} \mid r \in \mathbf{H}, k=\mathbf{1}, \ldots, n, i \in \mathbf{I}_{k d(k, r)}, d(k, r) \in \mathbf{M}_{i}^{9}\right\}$, $\overline{\mathbf{T}}_{r}=\max \left\{L_{i d(k, r)} / \underline{S}_{i}^{\vartheta} \mid r \in \mathbf{H}, k=\mathbf{1}, \ldots, n, i \in \mathbf{I}_{k d(k, r)}, d(k, r) \in \mathbf{M}_{i}^{\vartheta}\right\}$. As a result, the problem $\mathbf{B}_{1}(\lambda)$ is as follows:

$L(X, T, \lambda) \quad E(\lambda) \sum_{r=1}^{h} T_{r}+\sum_{i \in \mathbf{I}} \sum_{\vartheta=1}^{\gamma_{i}} f_{i}^{\vartheta}\left(X_{i}^{\vartheta}, \lambda\right) \rightarrow \min ;$

$X \in \mathbf{X}, T \in \overline{\mathbf{T}}_{0} ;$

$L_{i d(k, r)} / S_{i}^{\vartheta} \leq T_{r}, r \in \mathbf{H}, k=1, \ldots, n, i \in \mathbf{I}_{k d(k, r)}, d(k, r) \in M_{i}^{\vartheta}$,

where $E(\lambda)=\lambda E_{1}+(1-\lambda) E_{2}, g_{i j}(\lambda)=\lambda g_{1 i j}+(1-\lambda) g_{2 i j}$ and $f_{i}^{\vartheta}\left(X_{i}^{\vartheta}, \lambda\right)=\sum_{j \in J_{i}^{\vartheta}} g_{i j}(\lambda) / D_{i j}\left(x_{i j}\right)>0$.

In accordance with (Levin and Tanaev, 1998), if $\left(X^{*}(\lambda), T^{*}(\lambda)\right)$ is a solution of problem $\mathbf{B}_{1}(\lambda)$, then $X^{*}(\lambda)$ is a solution of problem $\mathbf{A}_{1}(\lambda)$. On the other hand, if $X^{*}(\lambda)$ is a solution of problem $\mathbf{A}_{1}(\lambda)$, then $\left(X^{*}(\lambda), \overline{\mathbf{t}}\left(X^{*}(\lambda)\right)\right)$ is a solution of problem $\mathbf{B}_{1}(\lambda)$.

Problem $\mathbf{B}_{1}(\lambda)$ can also be solved with the following two-level iterative decomposition scheme:

- at the lower level, cutting modes $X$ are optimized for fixed values of vector $T \in \overline{\mathbf{T}}_{0}$ of the takt times durations;

- at the upper level, the values of vector $T \in \overline{\mathbf{T}}_{0}$ are optimized.

The problem of the lower level is decomposed into $\sum_{i \in \mathrm{I}} \gamma_{i}$ of following independent problems for each vector $X_{i}^{\vartheta}$ of cutting mode parameters, $i \in \mathbf{I}, \vartheta=1, \ldots, \gamma_{i}\left(\operatorname{problems} \mathbf{B}_{i}^{\vartheta}(\lambda, T)\right)$ :

$f_{i}^{\vartheta}\left(X_{i}^{\vartheta}, \lambda\right) \rightarrow \min$,

$X_{i}^{\vartheta} \in \mathbf{X}_{i}^{\vartheta}$,

$1 / S_{i}^{\vartheta} \leq \sigma_{i}^{\vartheta}$,

where

$\sigma_{i}^{\vartheta} \quad \sigma_{i}^{\vartheta}(T)=\min \left\{T_{r} / L_{i d(k, r)} \mid r \in \mathbf{H}, k=1, \ldots, n, d(k, r) \in \mathbf{M}_{i}^{\vartheta}\right\}$,

$T \quad\left(T_{1}, \ldots, T_{h}\right)$ and $\lambda$ are fixed.

Let $X_{i}^{\vartheta}\left(\sigma_{i}^{\vartheta}, \lambda\right)=\left(S_{i}^{\vartheta}\left(\sigma_{i}^{\vartheta}, \lambda\right),\left(w_{i j}\left(\sigma_{i}^{\vartheta}, \lambda\right) \mid j \in \mathrm{J}_{i}^{\vartheta}\right)\right)$ be the solution and $\varphi_{i}^{\vartheta}\left(\sigma_{i}^{\vartheta}, \lambda\right)$ be the optimal value of the objective function of problem (11)-(13) for fixed values of $\sigma_{i}^{\vartheta}$ and $\lambda$. It is assumed 
that $\varphi_{i}^{\vartheta}\left(\sigma_{i}^{\vartheta}, \lambda\right)=\infty$ if inequalities (12)-(13) are incompatible for current $\sigma_{i}^{\vartheta}$. Then, the problem $\mathbf{B}_{11}(\lambda)$ of the upper level is as follows:

$\Psi(T, \lambda) \quad E(\lambda) \sum_{r=1}^{h} T_{r}+\sum_{i \in \mathrm{I}} \sum_{\vartheta=1}^{\gamma_{i}} \varphi_{i}^{\vartheta}\left(\sigma_{i}^{\vartheta}(T), \lambda\right) \rightarrow \min$,

$T \in \overline{\mathbf{T}}_{0}$,

where $\lambda$ is fixed.

It is obvious, if $T^{*}(\lambda)$ is a solution of this problem, then $X_{i}^{9}\left(\sigma_{i}^{9}\left(T^{*}(\lambda)\right), \lambda\right)$ is a solution of the corresponding problem $\mathbf{A}_{1}(\lambda)$.

For the special case of the problem $\mathbf{A}$ considered in (Levin et al., 2012), the techniques for problem $\mathbf{B}_{11}(\lambda)$ was proposed. They were based on reducing problem $\mathbf{B}_{11}(\lambda)$ to the search for a shortest path in a circuit-free digraph. This approach is not directly applicable to a more general case considered here. Thus, the proposed approach to solve problem $\mathbf{B}_{11}(\lambda)$ is based on the following properties of function $\varphi_{i}^{9}\left(\sigma_{i}^{9}, \lambda\right)$.

Since problem $\mathbf{B}_{i}^{9}(\lambda, T)$ is a parametric convex problem in variables $\ln S_{i}^{\vartheta}, \ln w_{i j}$ and $\ln \sigma_{i}^{\vartheta}$, function $\varphi_{i}^{\vartheta}\left(\sigma_{i}^{\vartheta}, \lambda\right)$ is nonincreasing on $\sigma_{i}^{\vartheta}$ in the segment $\left[\underline{\sigma}_{i}^{\vartheta}, \bar{\sigma}_{i}^{9}\right]=\left[1 / \bar{S}_{i}^{9}, 1 / \underline{S}_{i}^{\vartheta}\right]$ and convex on $\sigma_{i}^{\vartheta}$ in the segment $\left[\underline{\sigma}_{i 1}^{\vartheta}, \bar{\sigma}_{i}^{\vartheta}\right]$, where $\underline{\sigma}_{i 1}^{\vartheta}$ is the minimal value $\sigma_{i}^{\vartheta} \in\left[\underline{\sigma}_{i}^{\vartheta}, \bar{\sigma}_{i}^{\vartheta}\right]$ that $\varphi_{i}^{\vartheta}\left(\sigma_{i}^{\vartheta}, \lambda\right)<\infty$. So, for each function $\varphi_{i}^{\vartheta}\left(\sigma_{i}^{\vartheta}, \lambda\right), i \in \mathbf{I}, \vartheta=1, \ldots, \gamma_{i}$, its following piecewise linear approximation on the segment $\left[\underline{\sigma}_{i 1}^{\vartheta}, \bar{\sigma}_{i}^{\vartheta}\right]$ can be obtained:

$\varphi_{i}^{\vartheta}\left(\sigma_{i}^{\vartheta}, \lambda\right) \approx \max \left\{a_{i v}^{\vartheta}+b_{i v}^{\vartheta} \sigma_{i}^{\vartheta} \mid v=1, \ldots, \bar{v}_{i}^{\vartheta}\right\}$,

where $a_{i v}^{\vartheta}, b_{i v}^{9}$ and $\bar{v}_{i}^{9}$ are parameters of this approximation, $\bar{v}_{i}^{9}$ defines (in general) the accuracy of the approximation, $a_{i v}^{\vartheta}+b_{i v}^{\vartheta} \bar{\sigma}_{i}^{\vartheta}>0, b_{i v}^{\vartheta} \leq 0$, for all $i \in \mathrm{I}, \vartheta=1, \ldots, \gamma_{i}, v=1, \ldots, \bar{v}_{i}^{\vartheta}$.

Then, the resolution of $\mathbf{B}_{11}(\lambda)$ can be reduced to solving the following linear programming problem:

$L(T, \lambda, y) \quad E(\lambda) \sum_{r=1}^{h} T_{r}+\sum_{i \in \mathbf{I}} \sum_{\vartheta=1}^{\gamma_{i}} y_{i}^{\vartheta} \rightarrow \min$,

$L_{i d(k, r)} \sigma_{i}^{\vartheta} \leq T_{r}, r \in \mathbf{H}, k=1, \ldots, n, i \in \mathbf{I}_{k d(k, r)}, d(k, r) \in \mathbf{M}_{i}^{\vartheta}$,

$a_{i v}^{\vartheta}+b_{i v}^{\vartheta} \sigma_{i}^{\vartheta} \leq y_{i}^{\vartheta}, i \in \mathbf{I}, \vartheta=1, \ldots, \gamma_{i}, v=1, \ldots, \bar{v}_{i}^{\vartheta}$,

$\underline{\sigma}_{i 1}^{\vartheta} \leq \sigma_{i}^{\vartheta} \leq \bar{\sigma}_{i}^{\vartheta}, i \in \mathbf{I}, \vartheta=1, \ldots, \gamma_{i}$,

where sought variables of the problem are vectors $T=\left(T_{r} \mid r \in \mathbf{H}\right), y \quad\left(y_{i}^{\vartheta} \mid i \in \mathbf{I}, \vartheta=1, \ldots, \gamma_{i}\right)$ and

$\bar{\Sigma}=\left(\sigma_{i}^{\vartheta} \mid i \in \mathrm{I}, \vartheta=1, \ldots, \gamma_{i}\right)$.

If $\left(T^{*}, y^{*}, \bar{\Sigma}^{*}\right)$ is a solution of problem (17)-(20), then $X_{i}^{\vartheta}\left(\sigma_{i}^{\vartheta}\left(T^{*}\right), \lambda\right), i \in \mathbf{I}, \vartheta=1, \ldots, \gamma_{i}$ is a solution of problem $\mathbf{A}_{1}(\lambda)$ for given $\lambda$.

To obtain coefficients $a_{i v}^{\vartheta}, b_{i v}^{\vartheta}, \quad v=1, \ldots, \bar{v}_{i}^{9}$ of approximate function (16), values $\varphi_{i}^{\vartheta}\left(\sigma_{i}^{\vartheta}, \lambda\right)$ for some $\sigma_{i}^{\vartheta} \in\left[\underline{\sigma}_{i}^{\vartheta}, \bar{\sigma}_{i}^{\vartheta}\right]$ are to be found by the resolution of problem (11)-(13) for these $\sigma_{i}^{\vartheta}$ and $\lambda$.

Note some useful properties of problem (11)-(13):

a) if for some $\tilde{\sigma}_{i}^{9}$, inequalities (12)-(13) are incompatible, then they are also incompatible for all $\tilde{\sigma}_{i}^{9} \geq \sigma_{i}^{\vartheta} \in\left[\underline{\sigma}_{i}^{\vartheta}, \bar{\sigma}_{i}^{\vartheta}\right]$;

b) if $1 / S_{i}^{9}\left(\tilde{\sigma}_{i}^{9}, \lambda\right)=\sigma^{0}<\tilde{\sigma}_{i}^{\vartheta}$ for some $\tilde{\sigma}_{i}^{\vartheta} \in\left[\underline{\sigma}_{i}^{9}, \bar{\sigma}_{i}^{9}\right]$, then $X_{i}^{\vartheta}\left(\sigma_{i}^{\vartheta}, \lambda\right) \quad X_{i}^{\vartheta}\left(\tilde{\sigma}_{i}^{\vartheta}, \lambda\right)$ for all $\tilde{\sigma}_{i}^{\vartheta}<\sigma_{i}^{\vartheta} \in\left[\underline{\sigma}_{i}^{\vartheta}, \bar{\sigma}_{i}^{9}\right]$;

c) if $1 / S_{i}^{\vartheta}\left(\tilde{\sigma}_{i}^{\vartheta}, \lambda\right)=\tilde{\sigma}_{i}^{9}$ for some $\tilde{\sigma}_{i}^{9} \in\left[\underline{\sigma}_{i}^{\vartheta}, \bar{\sigma}_{i}^{9}\right]$, then $1 / S_{i}^{\vartheta}\left(\sigma_{i}^{\vartheta}, \lambda\right)=\sigma_{i}^{\vartheta}$ for all $\tilde{\sigma}_{i}^{\vartheta}>\sigma_{i}^{\vartheta} \in\left[\underline{\sigma}_{i 1}^{\vartheta}, \bar{\sigma}_{i}^{9}\right]$.

In general, problem (11)-(13) can be effectively solved with the use of well known methods of convex programming and of posinomial geometrical programming. From the property c), it follows that in many cases this problem can be solved as separable one.

Approximations (16) of the functions $\varphi_{i}^{\vartheta}\left(\sigma_{i}^{\vartheta}, \lambda\right)$ with necessary accuracy can be made at once or iteratively with the successive use of solutions of problem (17)-(20).

The considered approach to solve $\mathbf{B}_{11}(\lambda)$ can be used to approximate a solution of initial problem $\mathbf{A}$.

Let $a_{i v 0}^{\vartheta}, b_{i v 0}^{\vartheta}, \bar{v}_{i 0}^{9}$, and $a_{i v 1}^{\vartheta}, b_{i v 1}^{\vartheta}, \bar{v}_{i 1}^{9}$ be parameters of approximation (16) of functions $\varphi_{i}^{9}\left(\sigma_{i}^{\vartheta}, 0\right), \quad \varphi_{i}^{\vartheta}\left(\sigma_{i}^{9}, 1\right)$ respectively, $i \in \mathrm{I}, \vartheta=1, \ldots, \gamma_{i}$. Then, an approximate solution of initial problem $\mathbf{A}$ can be obtained as a solution of the following linear programming problem:

$E_{1} \sum_{r=1}^{h} T_{r}+\sum_{i \in \mathbf{I}} \sum_{\vartheta=1}^{\gamma_{i}} y_{i 1}^{\vartheta} \rightarrow \min$,

$E_{2} \sum_{r=1}^{h} T_{r}+\sum_{i \in \mathbf{I}} \sum_{\vartheta=1}^{\gamma_{i}} y_{i 0}^{\vartheta} \leq t_{0}$,

$L_{i d(k, r)} \sigma_{i}^{\vartheta} \leq T_{r}, r \in \mathbf{H}, k=1, \ldots, n, i \in \mathbf{I}_{k d(k, r)}, d(k, r) \in \mathbf{M}_{i}^{\vartheta}$,

$a_{i v 0}^{\vartheta}+b_{i v 0}^{\vartheta} \sigma_{i}^{\vartheta} \leq y_{i 0}^{\vartheta}, i \in \mathbf{I}, \vartheta=1, \ldots, \gamma_{i}, v=1, \ldots, \bar{v}_{i 0}^{\vartheta}$,

$a_{i v 1}^{\vartheta}+b_{i v 1}^{\vartheta} \sigma_{i}^{\vartheta} \leq y_{i 1}^{\vartheta}, \quad i \in \mathbf{I}, \vartheta=1, \ldots, \gamma_{i}, v=1, \ldots, \bar{v}_{i 1}^{\vartheta}$,

$\underline{\sigma}_{i 1}^{\vartheta} \leq \sigma_{i}^{\vartheta} \leq \bar{\sigma}_{i}^{\vartheta}, i \in \mathbf{I}, \vartheta=1, \ldots, \gamma_{i}$.

In this problem, the sought variables are vectors $T=\left(T_{r} \mid r \in \mathbf{H}\right), y_{0} \quad\left(y_{i 0}^{\vartheta} \mid i \in \mathbf{I}, \vartheta=1, \ldots, \gamma_{i}\right), \quad y_{1} \quad\left(y_{i 1}^{\vartheta} \mid i \in \mathbf{I}, \vartheta=1\right.$, $\left., \ldots, \gamma_{i}\right)$, and $\bar{\Sigma}=\left(\sigma_{i}^{\vartheta} \mid i \in \mathrm{I}, \vartheta=1, \ldots, \gamma_{i}\right)$.

If $\left(T^{*}, y_{0}^{*}, y_{1}^{*}, \bar{\Sigma}^{*}\right)$ is a solution of problem (21)-(26), then $X_{i}^{\vartheta}\left(\sigma_{i}^{\vartheta}\left(T^{*}\right), 1\right), i \in \mathbf{I}, \vartheta=1, \ldots, \gamma_{i}$ can be accepted as an approximate solution of the initial problem $\mathbf{A}$.

The accuracy of the solution obtained mainly depends on the impact of parameter $\lambda \in[0,1]$ on the value of functions $\varphi_{i}^{\vartheta}\left(\sigma_{i}^{\vartheta}, \lambda\right)$. 


\section{CONCLUSION}

The problem considered here appears for optimization of cutting modes when designing manufacturing processes for batch processing on multiple position machines with multispindle heads. Batches can contain parts of several types. The tools of each spindle head employed for different parts can differ. Each tool can perform different manufacturing operations for different parts but with the same cutting modes. In this paper, this complex optimization problem is formulated in terms of a geometric program. Then, a novel decomposition approach is suggested. The proposed techniques reduce the search for an approximate solution of this problem to a finite sequence of easier convex and linear programming sub-problems which can be efficiently solved by known methods.

\section{ACKNOWLEDGMENT}

This work has been partially supported by the joint project BRFFR Ф12ФП-001 and PICS CNRS n 6064.

\section{REFERENCES}

Agrawal, A.K., Bhardwaj, P. and Srivastava, V. (2011). Ant colony optimization for group technology applications. International Journal of Advanced Manufacturing Technology, 55 (5), 783-795.

Battaia, O., Dolgui, A., Guschinsky, N. and Levin, G. (2012a). A decision support system for design of mass production machining lines composed of stations of rotary and mobile table. Robotics and Computer Integrated Manufacturing, 28, 672-680.

Battaïa, O., Dolgui, A., Guschinsky, N. and Levin, G. (2012b). Optimal design of machines processing pipeline parts. International Journal of Advanced Manufacturing Technology, 63 (9-12), 963-973.

Cakir, M.C. and Gurarda, A. (2000). Optimization of machining conditions for multi-tool milling operations. International Journal of Production Research, 38 (15), 3537-3552.

Dolgui, A., Guschinsky, N. and Levin, G. (2006). A decomposition method for transfer line life cycle cost optimisation. Journal of Mathematical Modeling and Algorithms, 5 (2), 215-238.

Dolgui, A., Guschinsky, N., Levin, G. and Proth, J.-M. (2008a). Optimisation of multi-position machines and transfer lines. European Journal of Operational Research, 185, 1375-1389.

Dolgui A., Guschinsky N., Guschinskaya O., and Levin G. (2008b). Decision making and support tools for design of transmission systems. In: Encyclopedia of Decision Making and Decision Support Technologies, F. Adam, P. Humpreys (ed.), Information Science Reference, Hershey, NY, 1, 165-175.

Dolgui A., Guschinsky N., and Levin G. (2009a). A Design of DSS for Mass Production Machining Systems, The Bulletin of the Polish Academy of Sciences - Technical Sciences, 57 (3), 265-271.

Dolgui, A., Guschinsky, N. and Levin, G (2009b) Graph approach for optimal design on transfer machine with rotary table. International Journal of Production
Research, 47 (2), 321-341.

Dolgui, A., Guschinsky, N. and Levin, G. (2012). Enhanced mixed integer programming model for a transfer line design problem, Computers \& Industrial Engineering, 62 (2), 570-578.

Dolgui, A., and Proth, J.M. (2006). Les systèmes de production modernes, 2 volumes, Hermès/Lavoisier.

Halevi, G. (2003). Process and Operation Planning, Kluwer.

Hui, Y.V., Leung, L.C. and Linn, R. (2001). Optimal machining conditions with cost of quality and tool maintenance for turning. International Journal of Production Research, 39 (4), 647-665.

Ismail, N., Musharavati, F., Hamouda, A. and Ramli, A. (2008) Manufacturing process planning optimisation in reconfigurable multiple parts flow lines. Journal of Achievements in Materials and Manufacturing Engineering, 31 (2), 671-677.

Juan, H., Yu, S.F. and Lee, B.Y. (2003). The optimal cutting parameter selection of production cost in HSM for SKD61 tool steels. International Journal of Machine Tools and Manufacture, 43, 679-686.

Koren, Y., Heisel, U., Jovane, F., Moriwaki, T., Pritschow, G., Ulsoy, G. et. al. (1999). Reconfigurable Manufacturing Systems. CIPR Annals, 48 (2), 6-12.

Koren, Y. (2010). The Global Manufacturing Revolution: Product-Process-Business Integration and Reconfigurable Systems. John Wiley and Sons.

Levin, G.M. and Tanaev, V.S. (1978). Decomposition Techniques for Optimization of Design Solutions. Science and Engineering. Minsk, Belarus (In Russian).

Levin, G.M. and Tanaev, V.S. (1998). Parametric decomposition for optimization problems. Proceedings of the NAS of Belarus, Ser. of Physical-Mathematical Sci., 4, 121-131. (In Russian).

Levin, G., Rozin B., and Dolgui A. (2012). Optimization of Multi-tool Cutting Modes for Batch Manufacturing in Large Series Machining Environment, Proceedings of the 14th IFAC Symposium on Information Control Problems in Manufacturing (INCOM12), Bucharest, Romania, May 23-25, 2012, T. Borangiu, I. Dumitrache, A. Dolgui, F. Filip (Eds.), Elsevier Science, 2012, IFACPapersOnline.net (ISSN 1474-6670), p. 444-448.

Liang, M., Mgwatu, M. and Zuo, M. (2001). Integration of cutting parameter selection and tool adjustment decision for multi-pass turning. International Journal of Advanced Manufacturing Technology, 17, 861-869.

Mukherjee, I. and Ray, P.K. (2006). A review of optimization techniques in metal cutting processes. Computers \& Industrial Engineering, 50 (1), 15-34. 